\title{
Low Complexity Method to Estimate Co-channel Signals using an Antenna Array
}

\author{
Shishir. K. L. and K. V. S. Hari \\ Department of ECE \\ Indian Institute of Science \\ Bangalore, India. \\ e-mail: \\ shishireprotocol.ece.iisc.ernet.in \\ hari@ece.iisc.ernet.in
}

\begin{abstract}
The estimation of binary, co-channel digital signals in a mobile multipath time-varying environment has been developed in [1]. However, it is computationally expensive since it involves the Singular Value Decomposition (SVD) of large matrices. In this paper, a low-complexity variant for the estimation of cochannel binary signals has been developed, which uses Iterative Least Squares with Projection (ILSP) and Iterative Least Squares with Enumeration (ILSE) directly on the received data. The variant developed here exploits the structure in the signal matrix to reduce the complexity required for estimation of the signals. It has been found that though the algorithm is suboptimal, the Bit Error Rate(BER) performance is quite satisfactory, while the computational complexity is reduced to a great extent.
\end{abstract}

\section{INTRODUCTION}

The blind estimation of co-channel signals using an antenna array, has been an area of active research in the recent times. There have been studies on estimation of signals in a mobile environment, but with the channel assumed to be of single tap. These algorithms make use of some property of the transmitted signal to get an estimate of the channel without the use of training sequences. Algorithms that assume that the signals are of Constant Modulus(Analytical Constant Modulus Algorithm(ACMA)) [2], or that the signals have a Finite Alphabet(FA) property [3] or that digital signals are cyclostationary in nature, have been reported in the literature.

Later, the techniques used for the case of single tap channels, were also found to be suitable for application to the case of multipath channels, but with some amount of preprocessing. The blind estimation of digital signals in the presence of multipath, using the FA property, has been considered in [1]. However, the method proposed in [1] is computationally expensive. This is because, the matrices involved in the process are large and their decomposition involves a lot of computation. In this paper, a computationally efficient technique to implement the algorithm presented in [1] has been proposed. Using the proposed method, detection of the number of finite-alphabet signals arriving at an antenna array, can also be done.

In section II, the data model used, is described. Section-III gives a brief description of the existing method and the computational complexity required for this method. Then the proposed method is described in detail. Next, a specific example is considered to compare the computational complexity of the two

This project was sponsored by Nokia Research Center, Helsinki, Finland under the IISc-NOKIA MOU.

\author{
Risto Wichman \\ NOKIA Research Center \\ Helsinki, Finland \\ e-mail: \\ risto.wichman@nokia.research.com
}

methods. In section-IV, numerical simulations of the proposed method are presented, followed by a discussion of certain points in section-V and conclusions in section-VI.

\section{THE DATA MODEL}

Consider $d$ BPSK signals being received by a Uniform Linear Array (ULA) of $M$ sensors. Let $L$ be the channel length as sampled at the receiver. The signal received at the antenna array is a convolution of the transmitted signal with the Channel Impulse Response (CIR). Thus, the received signal can be represented as [1]

$$
\mathbf{x}(t)=\mathbf{H}(t) * \mathbf{s}(t)+\mathbf{n}(t)
$$

where $*$ denotes convolution,

$$
\begin{aligned}
& \mathbf{x}(t)_{M \times 1}=\text { output of the } M \text { sensors, } \\
& \mathbf{H}(t)_{M \times d}=\text { CIR matrix; } h_{i j}(t) \text { is the CIR } \\
& \text { from the } j \text { th signal to the } i \text { th sensor, } \\
& \mathrm{s}(t)_{d \times 1}=\text { signal vector, consisting of } \pm 1 \mathrm{~s}, \\
& \mathbf{n}(t)_{M \times 1}=\text { noise vector. }
\end{aligned}
$$

Sampling at the symbol rate at the receiver, taking $N$ snapshots, and writing the convolution in matrix form with the common channel length as $L$, we have,

$$
\begin{aligned}
\mathbf{X}= & \mathbf{H} \mathbf{S}_{L} \\
\mathbf{H}= & {[H(0), \cdots, H(L-1)]_{M \times d L} } \\
\mathbf{S}_{L}= & {\left[\begin{array}{ccccc}
\mathbf{s}_{o} & \mathbf{s}_{1} & \cdots & \mathbf{s}_{N-2} & \mathbf{s}_{N-1} \\
\mathbf{s}_{-1} & \mathbf{s}_{o} & \cdots & \mathbf{s}_{N-3} & \mathbf{s}_{N-2} \\
\mathbf{s}_{-2} & \mathbf{s}_{-1} & \cdots & \mathbf{s}_{N-4} & \mathbf{s}_{N-3} \\
\vdots & & & & \vdots \\
\mathbf{s}_{-L+2} & \mathbf{s}_{-L+3} & \cdots & \mathbf{s}_{N-L+1} \\
\mathbf{s}_{-L+1} & \mathbf{s}_{-L+2} & \cdots & \mathbf{s}_{N-L}
\end{array}\right]_{d L \times N} }
\end{aligned}
$$

It is necessary that $\mathbf{H}$ is of full column rank. Due to this, the number of antennas $M$ must be more than $d L$. This means that the number of antennas required turns out to be fairly large. However, oversampling the antenna outputs by a factor $P>1$ would help in reducing this constraint to $M P>d L$. When oversampling is used, the matrix $\mathbf{H}$ can be represented as

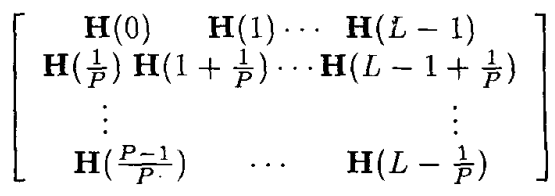


Further, by stacking shifted versions of the block rows of $\mathbf{X}$, this constraint can be reduced to $M P>d[1]$.

\section{METHODS FOR SIGNAL ESTIMATION}

\section{A. Complexity of existing method}

In [1], oversampling is done and $\mathbf{X}$ is manipulated by stacking shifted versions of its block rows. The number of block rows is denoted by $m . m$ is $\geq 2$ and the size of $\mathbf{X}$ increases with this. $A$ basis for the generator of the new $S_{L}$ (due to the stacked $X$ ) is obtained before using ILSP/ILSE.

The algorithm requires an SVD [4] of an $m M P \times(N-m)$ matrix in the first step. Then a subspace intersection algorithm is used to determine a basis for the generator of $\mathbf{S}_{L}$. The last step is the forcing of the FA property, for which Iterative Least Squares with Subspace Fitting(ILSF) [1] is used. The computational complexities of the three steps are shown in Table(I).

\section{B. Proposed Method for Signal Estimation}

The computational complexity of the above algorithm is significant. A computationally efficient method for estimation of signals is now proposed. Here, the requirement to find a basis for the generator of the block Toeplitz matrix $\mathbf{S}_{L}$, is dispensed with. Instead, ILSP/ILSE is applied directly on the received signal matrix with oversampling, i.e., $\mathbf{X}$ or after stacking shifted rows of $\mathbf{X}$, in order to ease the requirement on the number of sensors.

The FA algorithms, ILSP/ILSE solve the following Least Squares problem:

$$
\min _{\mathbf{H}, \mathbf{S}_{L}}\left\|\mathbf{X}-\mathbf{H} \mathbf{S}_{L}\right\|_{F}^{2}
$$

The matrices $\mathbf{H}$ and $\mathbf{S}_{L}$ which minimize Eq.(6) can be found through alternating projections. This forms one algorithm, called Iterative Least-Squares with Projection(ILSP). Starting from an initial estimate of $\mathbf{H}$ as, say $\mathbf{H}_{o}$ which can be taken as identity, the ILSP gives a coarse estimate of $\mathbf{H}$ and $\mathbf{S}_{L}$.

Using the property of the Frobenius Norm, Eq.(6) can be split so that the rninimization can be carried out columnwise. Now an enumeration over all possible $\mathbf{S}_{L}$ matrices with elements from the FA set can be carried out, so that the above cost function is minimized. This forms the second algorithm, ILSE.

It can be seen that $\mathbf{S}_{L}$ has a nice structure which can be made use of. The last $(d L-d)$ elements of the $n$th column of $\mathbf{S}_{L}$ are same as the first $(d L-d)$ elements of the $(n-1)$ th column. With this, we effectively need to estimate only the first $d$ rows of $\mathbf{S}_{L}$ and copy the last $(d L-d)$ elements from the previous columns. This means enumeration over only $2^{d}$ combinations of \pm 1 s. Di.e to this, the computational complexity is drastically reduced.

The above method works, only if we already have a good estimate of $S_{L}$ before using ILSE. This can be achieved by first using ILSF on the signal matrix $\mathbf{X}$. However, when ILSP is used to estimate the signals, there is an ordering and sign ambiguity in the estirnated signals as shown in [3]. First, the ordering and sign ambiguities have to be removed from the estimated signal matrix $\hat{\mathbf{S}}_{L}$ in order to obtain an ordered matrix which exhibits the block Toeplitz structure.
The ordering of $\hat{\mathbf{S}}_{L}$ can be done using a short labelling sequence. Consider the following relationship between the true signal matrix and the estimated signal matrix

$$
\hat{\mathbf{S}}_{L}=\mathbf{T} \mathbf{S}_{L}
$$

where $\tilde{\mathbf{S}}_{L}$ is the ordered matrix and $\mathbf{T}$ is a permutation matrix with its nonzero elements as \pm 1 . This is the ordering matrix. In the data model under consideration, $\mathbf{S}_{L}$ has more columns than rows. Thus,

$$
\mathbf{T}=\tilde{\mathbf{S}}_{L} \mathbf{S}_{L}^{\dagger}=\tilde{\mathbf{S}}_{L} \mathbf{S}_{L}\left(\mathbf{S}_{L} \mathbf{S}_{L}^{T}\right)^{-1}
$$

where $(.)^{\dagger}$ denotes pseudoinverse. Since the ordering matrix is a permutation matrix, it is non-singular and so can be inverted to obtain the ordered data matrix, as follows.

$$
\tilde{\mathbf{S}}_{L}=\mathbf{T}^{-1} \hat{\mathbf{S}}_{L}
$$

Thus, given a labelling sequence of all the co-channel signals, one can resolve the ambiguity. This is similar to the training sequence requirement for channel estimation. The assumption is that the same ordering matrix is valid for all symbols in the burst. However, the labelling sequence required is short and does not cause a large overhead on the data transmitted in a time-slot.

Here, the transmitted labelling sequence matrix has to be a wide matrix for Eq.(8) to hold. However, since $d L$ is the number of rows of the estimated signal matrix using the above channel model, the number of columns has to be more than $d L$. For example, it has been found through simulations, that for 2 signals with a channel of length $L=3$, a labelling sequence of length 10 , has to be used.

The matrix $\mathbf{T}$ obtained is first used to resolve ordering and sign ambiguities in $\hat{\mathbf{H}}$ and $\hat{\mathbf{S}}_{L}$. Eq.(7) can be used to remove the ambiguities in $\hat{\mathbf{S}}_{L}$ and Eq.(10) can be used to remove the ambiguities in $\hat{\mathbf{H}}$.

$$
\tilde{\mathbf{H}}=\hat{\mathbf{H}} \mathbf{T}
$$

While the requirement for the ordering of $\hat{\mathbf{S}}_{L}$ is evident, a similar requirement for $\hat{\mathbf{H}}$, is not so. It can be observed that an unordered $\hat{\mathbf{H}}$ matrix, when used with an ordered $\tilde{\mathbf{S}}_{L}$ results in the minimum norm being obtained for the wrong combination of \pm 1 s in a particular column of $\tilde{\mathbf{S}}_{L}$ when ILSE is used. In order to avoid this, ordering of the $\hat{\mathbf{H}}$ matrix also has to be done.

The ordered signal and channel matrices are now, $\tilde{\mathbf{S}}_{L}$ and $\tilde{\mathbf{H}}$ respectively.

Once the ambiguities are resolved, ILSE can be applied as in [1] to estimate only the new $d$ symbols in each column while the rest can be copied from the previous column. It must be noted here, that it is also necessary to determine the number of signals, $d$ for ILSE to be used.

\section{Summary of the estimation algorithm:}

- Get an estimate $\hat{\mathbf{H}}$ and $\hat{\mathbf{S}}_{L}$ using ILSP and resolve ordering and sign ambiguities. 
- Run ILSE. For this, copy the repeated first $(d L-d)$ elements from $(k-1)$ th column to the last $(d L-d)$ elements of the $k$ th column and use ILSE to estimate only the remaining $d$ symbols. Repeat for all snapshots.

Further, if Akaike Information-theoretic Criterion(AIC) is used to first determine the dimension of the signal subspace of $\mathbf{X}$, then a very simple method after ILSP gives the number of signals received.

\section{Computational complexity}

An illustrative example is now considered in order to compare the computational requirements of the method in [1] and the one proposed here.

Example: Let $d=2, M=2, P=2$, the minimum stacking parameter, $m=2$, and $N=50$. Table(I) summarizes the computations required by the two methods in general, while Table(II) gives the computations for the example considered.

It can be seen that the computational complexity of the proposed algorithm is an order of magnitude less than that of the method in [1].

\section{SIMULATION EXAMPLE}

The following situation has been considered for simulation:

- Number of signals $d=2$.

- Multipath channel length $L=3$.

- Oversampling factor, $P=2$.

- $S N R=5 d B$. Noise is considered to be Additive White Gaussian and generated as in [1].

- In these simulations, only oversampling has been incorporated, while the ordering of the $\mathbf{X}$ matrix has NOT been incorporated. So, the number of sensors can be further reduced for the same BER performance.

The performance measure considered is the Bit Error Rate for different Signal to Interference Ratios(SIR). SIR is defined as the ratio of the norms of the channels corresponding to the 2 signals, for a particular source-sensor pair.

Fig.(1) shows a plot of BER vs SIR for 5 sensors as a function of the number of snapshots. Fig.(2) shows the plot of BER vs SIR for different number of sensors with the number of snapshots being 80 . We can see that the BER reduces either if the number of snapshots is increased, or if the number of sensors is increased, with increasing SIR. The effect of the number of sensors is seen to be more than the effect of the number of snapshots on the BER. Thus, with more number of sensors used, better performance can be obtained.

\begin{tabular}{|l|l|}
\hline Method & General \\
\hline Existing & $\begin{array}{l}3(m M P)^{2}(N-m)+10(m M P)^{3} \\
+O\left(d^{2}(L+m)^{4} N\right)+2 N d^{2}\end{array}$ \\
\hline Proposed & $O(N M d L)+O\left(2^{d} N M d\right)$ \\
\hline \hline
\end{tabular}

COMPUTATIONAL COMPLEXITY OF THE ALGORITHM IN [1] AND THE PROPOSED METHOD FOR THE GENERAL CASE.

\begin{tabular}{|l|l|}
\hline Method & For the Example \\
\hline Existing & $14144+O(125000)+400$ \\
\hline Proposed & $O(3000)+O(4000)$ \\
\hline
\end{tabular}

COMPUTATIONAL COMPLEXITY OF THE ALGORITHM IN [1] AND THE PROPOSED METHOD FOR THE EXAMPLE WITH $M=2, P=2, m=2$, $N=50, d=2, L=3$.

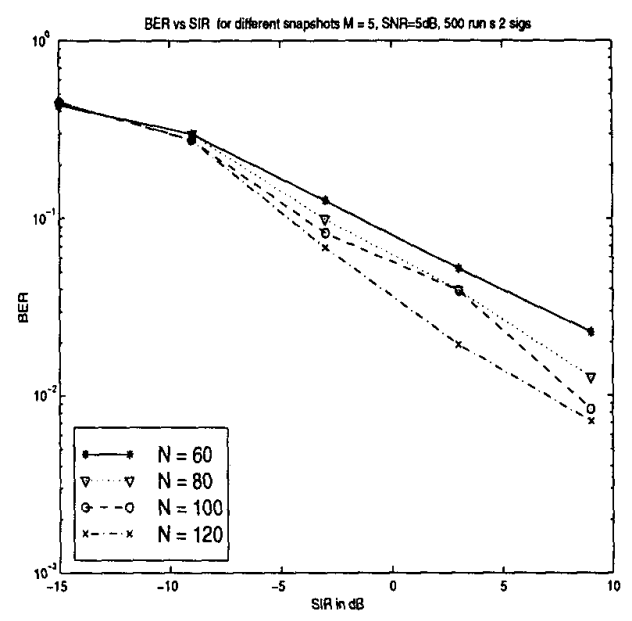

Fig. 1. BER vs SIR for a 5 sensor array receiving 2 BPSK signals over channels of $L=3$, as a function of the number of snapshots. The oversampling factor used is $P=2.500$ independent trials have been carried out for each N. $S N R=5 \mathrm{~dB}$.

\section{DISCUSSION}

There are certain issues in the above low-complexity algorithm, that need mention.

Since the rearrangement of $\mathbf{X}$ has not been considered here, the number of sensors is more. If the rearrangement is also done, the number of sensors can further be reduced by a factor equal to the number of block rows of $\mathbf{X}$ that are used.

The reduction in computational complexity, as has been seen, results mainly from the fact that the structure of $\mathbf{S}_{L}$ has been exploited. However, since the last $(d L-d)$ elements of a particular column are a replica of the first $(d L-d)$ elements of the previous column, (which means that the result of ILSP is used directly), the error is bound to be more than that obtained, if ILSE were used on the complete matrix.

Another issue is that, if there is an error in the first column itself, it tends to percolate to successive columns also. However, such drastic errors occur only at very low SIRs, which are generally not encountered in practice. At higher SIRs, the occurance of such errors is possible, but are so rare that the performance of the method is not affected much in the statistical sense.

\section{CONCLUSION}

A simple method of reducing computation has been proposed in the context of estimating finite-alphabet signals received by an antenna array in a multipath environment. It has beer shown through simulations that the BER performance of the method 


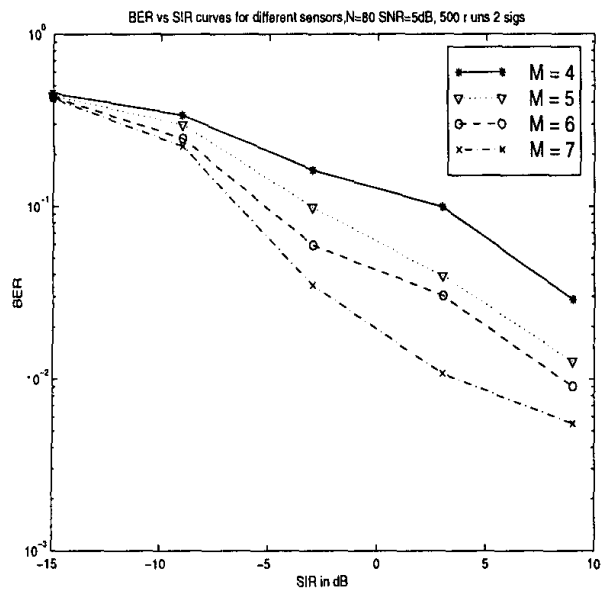

Fig. 2. BISR vs SIR with $\mathrm{N}=80$, as a function of number of sensors for 2 BPSK signals received over channels of $L=3$. The oversampling factor used is $P=2$. 500 independent trials have been carried out for each N. SNR = $5 \mathrm{~dB}$.

is satisfactory while a significant saving in computation can be obtained. A performance analysis of this algorithm may suggest methods to fine tune its performance further.

\section{REFERENCES}

[1] A. J. van der Veen, S. Talwar and A. Paulraj, "A subspace approach to blind space-time signal processing for wireless communication systems, " IEEE Trans. Signal Processing, vol. 45, pp. 173-190, January 1996.

[2] A.J. van der Veen, and A. Paulraj, "An analytical constant modulus algorithm," IEEE Trans. on Signal Processing, vol.44, pp. 1136-1155, May 1996.

[3] S. Talwar, M. Viberg, and A. Paulraj, "Blind separation of co-channel digital signals using an antenna array, Part I: Algorithms," IEEE Trans. Signal Processing, vol. 44, pp. 1184-1197 May 1996.

[4] G. H. Golub and C. L. Van Loan, Matrix Computations, Johns Hopkins University Press, 1987.

[5] M. Wax and T. Kailath, "Detection of signals by information theoretic criteria," IEEE Trans. on ASSP, vol. 33, pp. 387-392, April 1985. 